\title{
A Moodle-Based E-Syllabus with Database Activity Module
}

\author{
Yoshikazu Asada *, Hitoaki Okazaki *, Naohiro Sata *, \\ Hiroshi Kawahira *, Shinichi Yamamoto *, Yasushi Matsuyama *
}

\begin{abstract}
Institutional research is an essential topic in the medical education field. To achieve global standards, curriculum mapping and competency assessments based on the model core curriculum (MCC) and diploma policies (DP) must be discussed. This study investigated initial practices using a Moodle-based e-syllabus to gather and analyze curriculum information. Although several classes had some MCC elements, the results showed that others had no such connections. Since the Japanese MCC is defined as "a common, national, systematically organized curriculum taught in each medical faculty/university," it is crucial that each MCC element be fulfilled throughout the entire curriculum. Although the Moodle-based e-syllabus needs to be improved in some ways, especially in ease of use, the data integration and analytics aspects were better than those of the previous Excel-based syllabus.
\end{abstract}

Keywords: E-syllabus, Competencies, Curriculum Mapping, Medical Education, Model Core Curriculum, Moodle

\section{Introduction}

\subsection{IR and LMS in medical education}

Institutional research (IR) has become an important topic in medical education, mainly due to the need for program evaluations based on global standards [1][2], making the process of gathering data for assessing and evaluating the educational system essential. To this end, learning management systems (LMSs) are an important tool. Although a given LMS is mainly designed to support learning, some of the data gathered are useful for IR. In fact, the following statements can be found in the World Federation for Medical Education's global standards [1]:

- The medical school must formulate and implement a policy which addresses effective and ethical use and evaluation of appropriate information and communication technology. (Basic 6.3.1)

\footnotetext{
* Jichi Medical University, Tochigi, Japan
} 
- Effective and ethical use of information and communication technology would include use of computers, cell/mobile telephones, internal and external networks and other means as well as coordination with library services. The policy would include common access to all educational items through a learning management system. (Annotations in Area 6.3)

LMSs are also useful for assessing and evaluating education, and the global standards discuss LMS in the assessment context as follows [1]:

- use a wide range of assessment methods and formats according to their "assessment utility." (Basic 3.1.3)

- The medical school should incorporate new assessment methods where appropriate. (Quality development 3.1.2)

- The medical school must establish and apply a mechanism for programme evaluation that addresses student progress. (Basic 7.1.3)

It is therefore essential to implement LMSs in both education and IR.

\subsection{Curriculum mapping and the model core curriculum}

Area 2 of the global standards requires curriculum design to include [1]:

- ... a statement of the intended educational outcomes (cf.1.3), the content/syllabus (cf. 2.2-2.6), learning experiences and processes of the programme. (Annotations in Area 2.1)

In Japan, the model core curriculum (MCC) is defined by the Japanese Ministry of Education, Culture, Sports, Science and Technology [3]. MCC is defined as "a common, national, systematically organized curriculum taught in each medical faculty/university and which accounts for approximately $2 / 3$ of a total learning time." In other words, the remaining $1 / 3$ of the total learning time is designed by each medical institution and may thus include other topics, such as liberal arts education and advanced medical technology that uses artificial intelligence (AI).

The MCC comprises seven domains. The first concerns the "Basic Qualities and Abilities Required of a Physician" and includes nine elements, including "professionalism" and "medical knowledge and problem-solving ability." These nine elements are vertically connected to the competencies outlined in the postgraduate clinical training achievement objectives. Each medical institution has its own diploma policies (DP) for assessing student competencies, but these nine elements are consistently part of the evaluation process. Other MCC domains are also connected to postgraduate clinical training, including "Approaches from signs and symptoms/pathophysiology" and "Clinical reasoning." Thus, the MCC constitutes one of the bases for curriculum mapping in the medical education field.

Table 1 shows the taxonomy of the Jichi Medical University (JMU) syllabus. There are nine areas; the first is elective while the others are compulsory. The seventh area includes bed-side learning. Since JMU takes more than two years for bed-side learning, the required credits of this area are larger than those of other areas. 
Table 1: The taxonomy of JMU syllabus

\begin{tabular}{ccc}
\hline Domains & Theme & Required Credits \\
\hline $\mathbf{L 1}$ & General Education (Elective) & 10 \\
\hline $\mathbf{L 2}$ & General Education (Compulsory) & 25.7 \\
\hline L3 & Basic Medical Sciences & 45.75 \\
\hline $\mathbf{L 4}$ & Community Medicine & 7.9 \\
\hline L5 & Systematic Lecture (Basic Clinical Medicine) & 42.3 \\
\hline L6 & Social Medicine & 12.1 \\
\hline L7 & Clinical Medicine 1 (including bed-side learning) & 106.2 \\
\hline L8 & Clinical Medicine 2 (such as palliative care) & 8.4 \\
\hline L9 & Integrated Review & 24.7 \\
\hline
\end{tabular}

This study aimed to assess Moodle as a tool for conducting IR, especially for investigating curriculum mapping and competency assessments.

\section{Methods: Developing E-syllabus with Moodle}

\subsection{LMS for IR}

LMSs such as Moodle [4] and manaba [5] are already used in Japan. Moodle is a free and opensource system that is widely used throughout the world. Moodle features many free plugins, some of which enable users to export data directly from the Moodle database (e.g., MySQL, MariaDB, and PostgreSQL) [6][7]. These plugins are used for IR; they are also implemented in learning analytics [8]. On the other hand, some commercial-based LMSs contain native data-exporting features or have additional export costs. As such, this study focused on Moodle as an IR tool.

\subsection{Developing the e-syllabus using Moodle}

Information about objectives, assessment, and faculties was needed to conduct IR in each class, along with competencies. The global standards present the following requirements [1]:

- The medical school must have a programme of routine curriculum monitoring of processes and outcomes. (B 7.1.1)

- Programme monitoring would imply the routine collection of data about key aspects of the curriculum for the purpose of ensuring that the educational process is on track and for identifying any areas in need of intervention. The collection of data is often part of the administrative procedures in connection with admission of students, assessment and graduation. (Annotations in Area 7.1) 
Based on this information, LMSs such as Moodle were considered appropriate for integration with the e-syllabus, which includes curriculum information and online learning contents for data collection.

Although there were no basic modules or plugins for the e-syllabus, the database activity (DB) feature was offered as a standard Moodle module. This could be used to create an e-syllabus [9]. The DB feature is defined as follows: "The database activity module allows the teacher and/or students to build, display and search a bank of record entries about any conceivable topic. The format and structure of these entries can be almost unlimited, including images, files, URLs, numbers and text amongst other things" [10]. The DB feature offered an acceptable way to create the e-syllabus since it collected text-based information such as class name, objectives, class schedule, and office hours.

Note that it is important to consider the user permissions required to edit the syllabus data on Moodle. The default DB module enables teachers to show and edit all data. Thus, if all faculty members have a "teacher" role, all syllabus data are visible. In this case, faculty members have to search for and choose their own syllabus for editing. Since they can accidentally edit or delete all other data, careful operation of Moodle is needed. It can be stressful for users.

To avoid this situation, the feature that allows DB entries to be approved was used. Unapproved data are visible to all teachers. However, it is visible only to the "student" role who input the data. If all syllabus data are set to "not approved," faculty members with "student" roles who want to edit the syllabus can view only their own data. Thus, all faculty members were entered into the syllabus editing course in the "student" role.

DB modules have several fields where information can be entered, including check boxes and free-form text areas. The free-form text has three input types in Moodle: HTML format, Moodle auto-format, and plain text. Since the university uses both paper-based and web-based syllabi, the data should be exportable without HTML tags, allowing paper-based syllabi to be created. In the default setting of Moodle, the format is set to "HTML." To use plain text, the format must be chosen in each separate editor area; otherwise, the inputted texts are saved as HTML will be displayed without formatting. However, it is not reasonable to require each faculty member to choose plain text every time they fill a field.

To solve this problem, a JavaScript DB module was applied to the input form and view options. The module allows "plain text" to be automatically chosen in the specific text fields. Although it was not possible to automatically choose all fields as each one had a numbered ID, specifying the range of fields based on their IDs worked correctly, as shown in Figure 1. This code is based on "SetMoodlePlainTextEditMode.user.js" which is opened in GitHub [11]. 


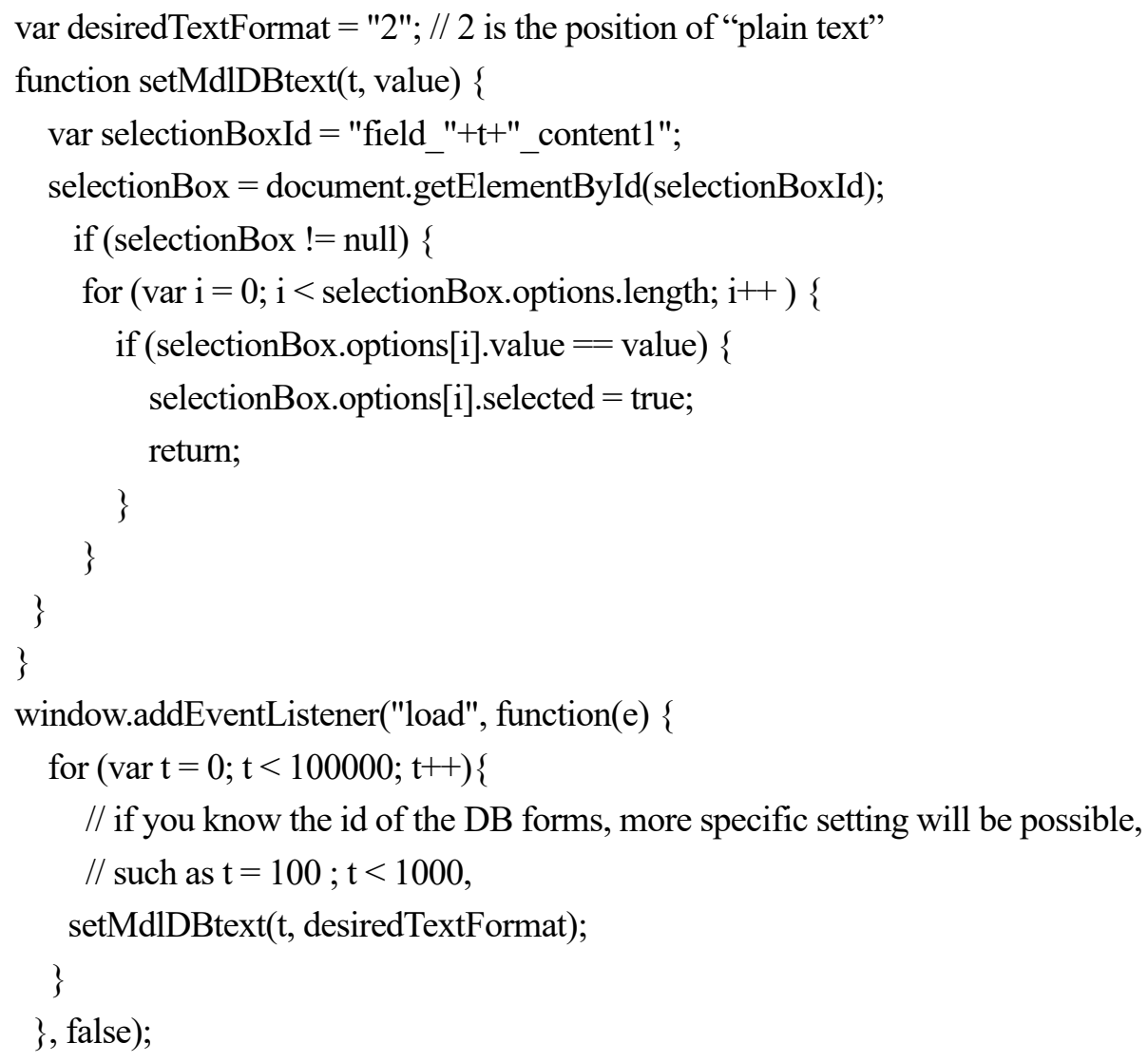

Figure 1: JavaScript template for automatically setting the text-style.

\subsection{Setting up competencies using Moodle}

Moodle contains a competencies-based education feature [12] in which competencies are defined as tree structures, meaning that more than one competency can be defined. Pre-defined competencies are also connected to each course and course activity. This study established two competencies through Moodle. The first was based on the MCC, while the second was based on the DP used at the author's university. Figure 2 shows the structure of competencies on Moodle. Although Moodle did not contain a feature for exporting whole connections between competencies and courses, it allowed data to be exported directly from the Moodle database via plugin. 


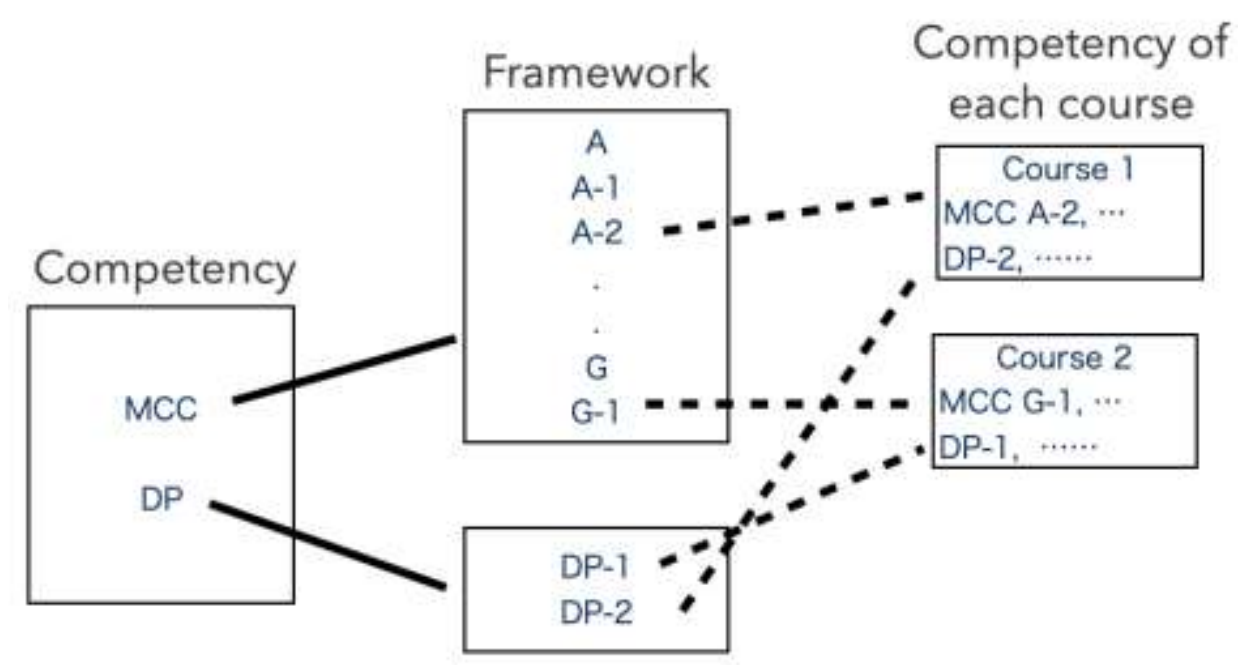

Figure 2: The structure of competencies on Moodle

\section{Methods: Exporting the Data from e-syllabus}

Although it is possible to set competencies for each class in Moodle, there are no features that allow the sitewide usage of competencies to be exported. Since the competency data are stored in the table, such as prefix_competency and prefix_competency_framework, joining these tables enables the aggregated data of competency usage. Figure 3 shows the SQL code for obtaining the total sitewide count of competencies [7]. In Figure 3, "Code" refers to the ID number of each competency. In this study, the dates of the 2020 syllabus were used. After exporting the data as an Excel or CSV file, it was visualized with Tableau - a widely used business intelligence tool for interactive data visualization which has previously been used in the area of healthcare [13].

\footnotetext{
SELECT crsc.name AS Course, c.idnumber AS Code, c.shortname AS Competency, count(crs.id) AS Count

FROM prefix_competency AS c

JOIN prefix_competency_framework AS cf ON cf.id = c.competencyframeworkid LEFT JOIN prefix_competency_coursecomp AS cc ON c.id= cc.competencyid LEFT JOIN prefix_course AS crs ON crs.id = cc.courseid LEFT JOIN prefix_course_categories AS crsc ON crsc.id = crs.category
}

Figure 3: SQL for aggregating the number of courses with competencies 


\section{Results}

\subsection{DB-based e-syllabus}

The DB has two layouts: a list view and a single view. Table 2 shows the list view style, which is intended for class indexing. Respective single-view pages are opened when users click class names. Single-view pages show general class information such as goals, objectives, assessment methods, class schedules, and textbooks.

Table 2: The style and the sample of the list view of DB based syllabus

\begin{tabular}{cccc}
\hline Domain & Title & Years & Link \\
\hline $\mathbf{L 2}$ & Introduction to Medicine & 1 st & Detailed page \\
\hline L3 & Anatomy & 1 st & Detailed page \\
\hline$\ldots$ & $\ldots$ & $\cdots$ & $\ldots$ \\
\hline
\end{tabular}

The DB-based e-syllabus is set on the top page of Moodle. When a class has its own Moodle courses, the student syllabus includes links to the page, created using the relevant course ID number. Officially, links to Moodle courses are based on IDs such as

moodle/course/view.php?id=1254.

These IDs are automatically added in the order that courses are created. However, since some classes did not have their own courses, it was difficult to automatically connect each syllabus and course.

Alternatively, a URL link based on the relevant course ID number was used. Each Moodle course has an optional course ID number that users can also refer to when accessing the course. For example, this could be:

moodle/course/view.php?idnumber=2019_M_L1262-2.

These course ID numbers are freely defined by site administrators. Thus, direct links can easily be made from the e-syllabus to their respective courses.

\subsection{The number of MCC and DP}

Both the MCC- and DP-based competencies were established within Moodle. The MCC-based competencies contained 467 elements (Table 3), while the DP-based competencies contained 41 elements (Table 4). 
Table 3: Numbers of MCC-Based Competencies Elements

\begin{tabular}{cccc}
\hline $\begin{array}{c}\text { Domains (sub } \\
\text { domains) }\end{array}$ & $\begin{array}{c}\text { Total MCC ele- } \\
\text { ments }\end{array}$ & $\begin{array}{c}\text { Total classes con- } \\
\text { nected to MCC }\end{array}$ & $\begin{array}{c}\text { Total number of elements with- } \\
\text { out any connections to classes }\end{array}$ \\
\hline $\mathrm{A}(9)$ & 26 & 114 & 3 \\
\hline $\mathrm{B}(4)$ & 16 & 37 & 3 \\
\hline $\mathrm{C}(5)$ & 53 & 40 & 34 \\
\hline $\mathrm{D}(15)$ & 186 & 125 & 26 \\
\hline $\mathrm{E}(9)$ & 51 & 52 & 62 \\
\hline $\mathrm{F}(3)$ & 75 & 25 & 53 \\
\hline $\mathrm{G}(4)$ & 60 & 16 &
\end{tabular}

Table 4: Numbers of DP-Based Competencies Elements

\begin{tabular}{ccc}
\hline Domains & Total DP elements & Total classes connected to DP \\
\hline I & 6 & 47 \\
\hline II & 13 & 197 \\
III & 6 & 41 \\
\hline IV & 16 & 90
\end{tabular}

The MCC contained some subdomains. For example, domain A consisted of nine subdomains, while domain B consisted of four subdomains. Figure 4 shows the results of each subdomain, including elements without any connections to classes. Since L1 is an elective course, the results focused on L2 - L9, which are compulsory.

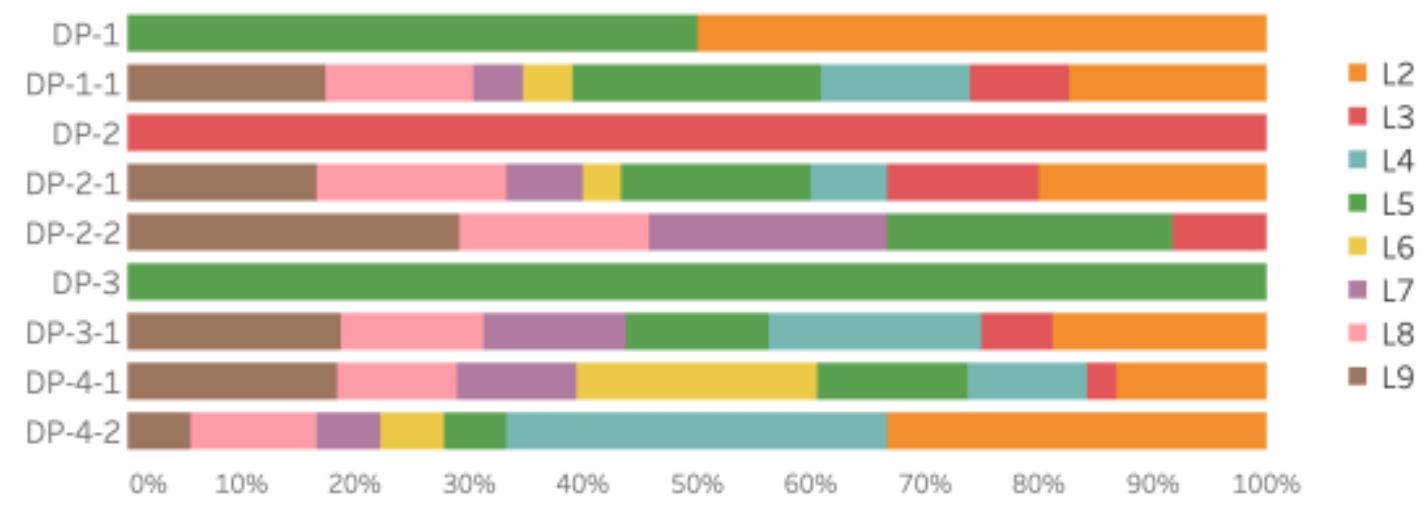

Figure 4: The mapping results of JMU diploma policies and syllabus

Similarly, Figure 5 shows the percentage of MCC and syllabuses. The MCC contained some subdomains. For example, domain A consisted of nine subdomains, while domain B consisted of four subdomains. Figure 5 shows the results of each subdomain, including elements without any connections to classes. As above, since L1 is an elective course, the results focused on L2 L9, which are compulsory. 
The blue bar (NA) shows the percentage of elements that had no connections to classes. For example, all elements of subdomain A-1 have some connections, while about $50 \%$ of elements had no connections in subdomain B-2. None of the elements in subdomain D-11 and G-1 had connections to classes.

Some MCC subdomains had no connections to classes. As shown in Figure 5, items D-11 and G-1 revealed values of $100 \%$, meaning that no classes treated them as objectives. In addition, none of the $\mathrm{D}$ and $\mathrm{G}$ subdomains had connected objectives.

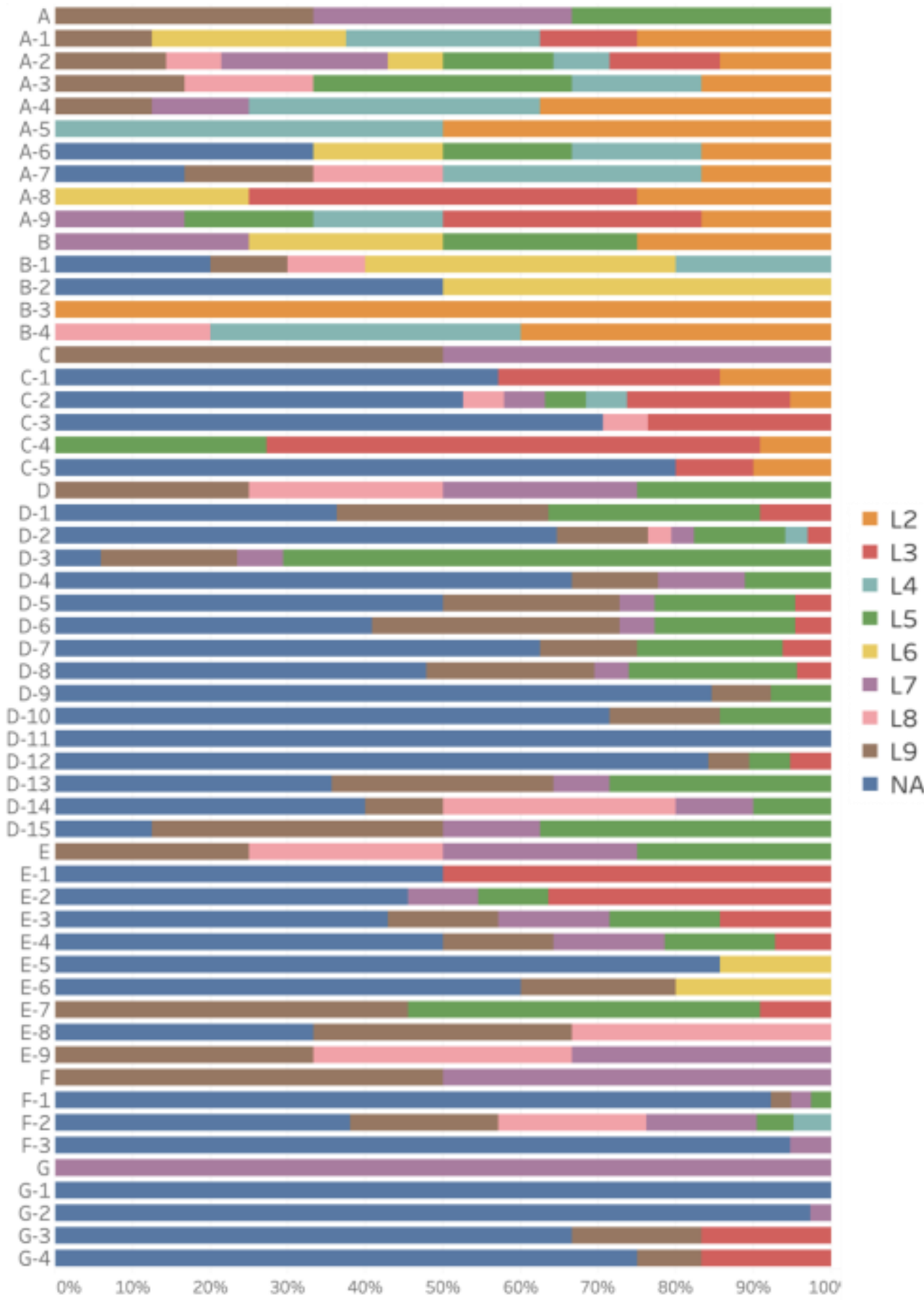

Figure 5: The mapping results of MCC and JMU syllabus 
Figure 6 shows the relationships between MCC and DP. In each column, circle size indicates the number of subjects. For example, there is only one subject that includes MCC A and DP 1, and 13 that include MCC A and DP 2-2. This shows that DP 2-1 and DP 2-2 have a wide connection to MCC. Since DP 2-1 and DP 2-2 are about the knowledge of basic medicine and clinical medicine, there are many related subjects in the syllabus.

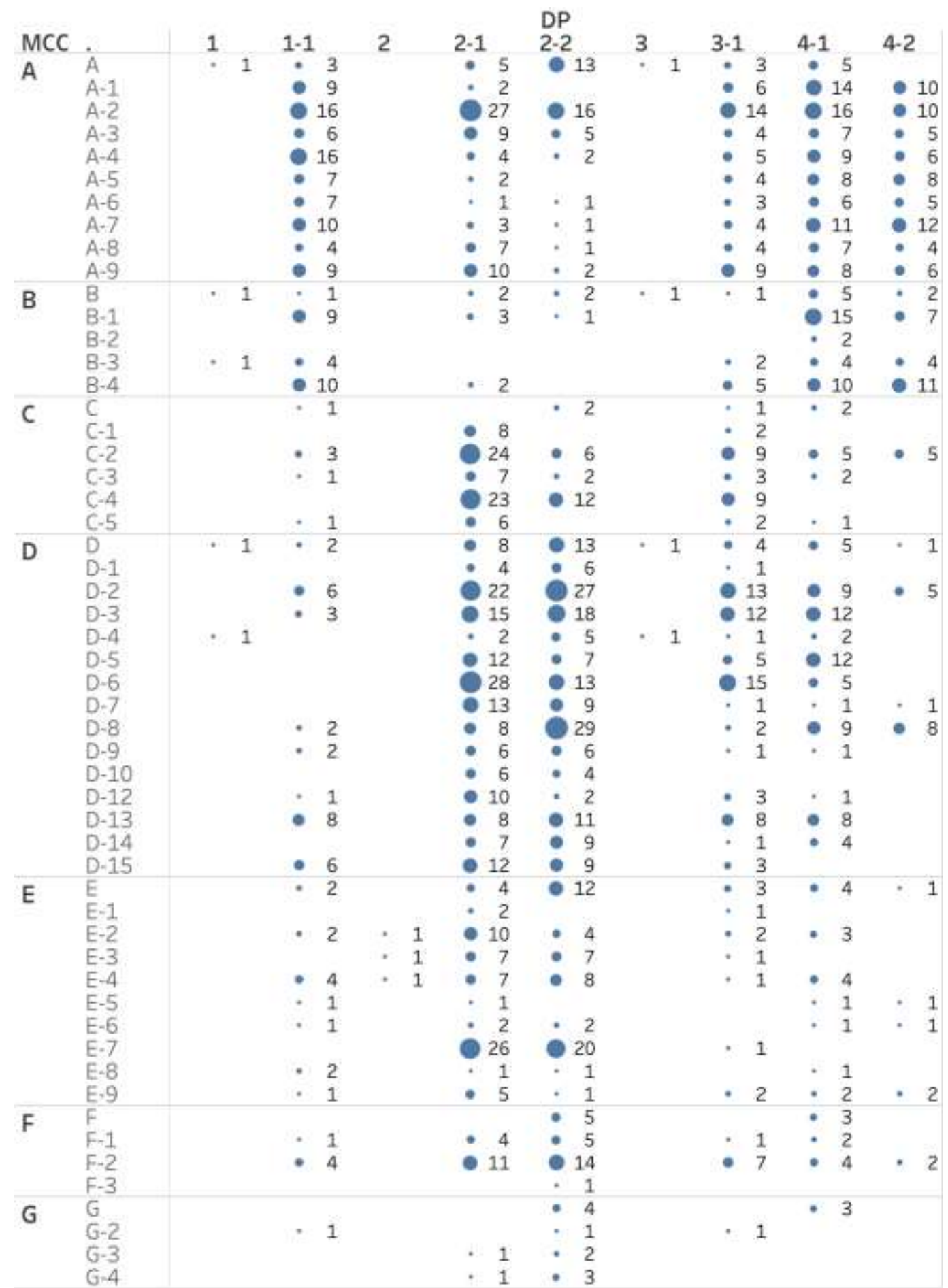

Figure 6: The relationships between MCC and DP 


\section{Discussion}

\subsection{DB-based e-syllabus}

Although this was the first attempt at importing data from the Excel syllabus used in previous years, almost all data were imported correctly. In 2020, due to COVID-19, almost all classes have their own Moodle courses, both lecture-based classes and bed-side learning, which will continue for some time [14]. Since each e-syllabus has a link to each course, Moodle should function as a learning and teaching portal site for students and faculties.

However, there are three main areas should be improved.

First, the style of data. Some Excel data were contained in "merged cells," which created problems when importing. Since the whole data was imported to Moodle after manual adjustments, the new version of the syllabus data worked well. However, these types of data processing are sometimes problematic for importing and exporting data. In fact, some universities, including JMU, use both LMS and grade management systems. Although LMS such as Moodle can export data in various styles, such as CSV, grade management systems may not have features to import or export the data freely. Thus, faculties must change or combine the data for using both systems. Although this is not the only problem involved with an e-syllabus, the smooth data connection is an essential part of IR.

Second, it is somewhat difficult to input and display each class schedule. The syllabus schedule contains four types of information, including date, time, content, and faculty name. The DB requires individual fields for each information type per class. For example, if there are 80 anatomy classes, then the DB requires 320 input fields. Figure 7 shows the input form for the schedule. This is almost the same as with the Excel-based format. In fact, it is more inconvenient to use the form since users cannot cut and paste the line.

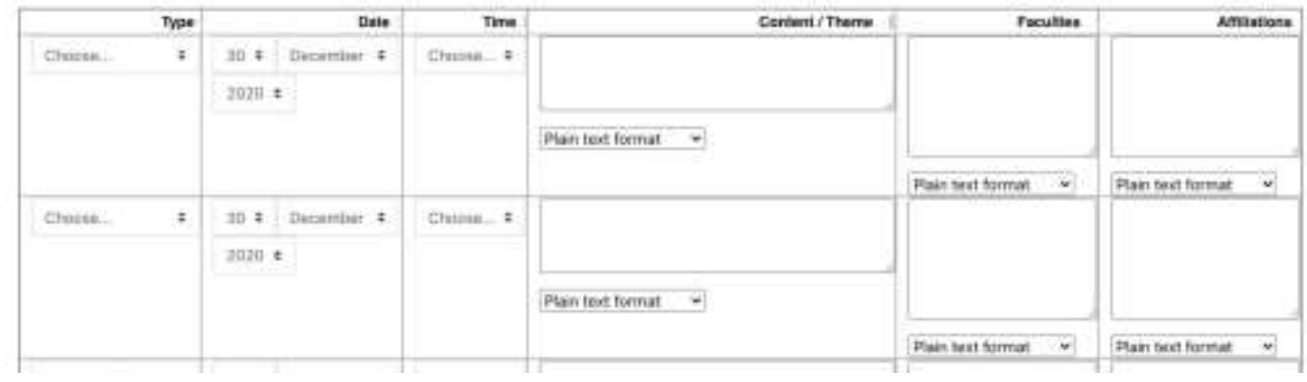

Figure 7: Input forms for course schedules

One way to improve this is by using the Moodle calendar feature. Currently, only monthly and daily views are available in Moodle, with no weekly view. Usability will improve if the weekly view format is developed so that faculty members can input their class schedules from other systems, such as Google Calendar. 
The third issue concerns the connections between activities and competencies in each class. Moodle can not only connect the classes to competencies, but can also connect the competencies to activities such as quizzes and forums. Currently, only a few courses contain unique quizzes and other activities. However, as noted before, since there has been some shift to online-based learning due to the COVID-19 outbreak, an increasing number of courses are implementing their own learning activities. If these activities will eventually be connected to the competencies, then it will be possible to conduct assessments based on the competencies.

\subsection{Competencies}

Some MCC subdomains had no connections to classes. As shown in Figure 6, items D-11 and G-1 revealed values of $100 \%$, meaning that no classes treated them as objectives. In addition, none of the $\mathrm{D}$ and $\mathrm{G}$ subdomains had connected objectives.

Domain D was listed as the "Normal Structure and Function, Pathophysiology, Diagnosis, and Treatment of Each Organ System of the Human Body." Notably, the "normal structure of each organ system of the human body" was nearly equal to the objectives outlined for anatomy. In fact, the syllabus data from the previous year had a certain limitation in which each class contained a maximum of three elements from the MCC; most important objectives were thus missing from the anatomy class data. Therefore, most elements connected to the anatomy were omitted. Importantly, the Moodle based e-syllabus does not limit the number of competencies for each class. This will reduce the number of elements with no connections to classes.

On the other hand, domain G was the "Clinical Clerkship." Since there were no electronic data for the clinical clerkship syllabus, only the syllabus for the lectures and laboratory courses were included in this study. For this reason, almost all of domain $\mathrm{G}$ was omitted. Because the new syllabus includes both lectures and clinical clerkships, competency information will be gathered correctly.

Figure 6 shows that there were only a few classes related to DP1, 2, and 3, resulting from the problematic user interface. Since DP1, 2, and 3 are the top DP categories, they should not be selected. For example, if someone selected DP2, we cannot know whether they refer to DP2-1, DP2-2, or both. However, DP1, 2 and 3 were unintentionally set as selectable. Although few classes selected these, the issue should be addressed in future to enable more accurate analysis.

\subsection{Further improvement}

There are some points that should be improved in the proposed system. First, the e-syllabus based on the DB. While the original URL with Moodle ID numbers works correctly from web browsers, such as Edge and Safari, it did not work in the Moodle Mobile App [15]. When users try to access the URL from the app, the default web browser opens automatically. Although the link itself worked correctly, this was inconvenient for users. This continues to be an issue in the newest Moodle Mobile App (3.9.3); therefore, further updates by Moodle HQ are needed.

The second point concerns the connection between each e-syllabus and course schedule. Since the date information is entered into Moodle through text-based data, there are no connections or direct links from the syllabus to activities or resources. One way to solve this problem is to use the calendar feature of Moodle, which could include dates for each activity, such as quizzes and forums. However, the displayed date only connects to the activities, not the topics. Thus, 
faculty members must create some activities beforehand to set the syllabus schedules for a Moodle calendar. Since almost all classes made Moodle courses and set activities in 2020 due to COVID-19, importing the contents to next year courses would be helpful in setting date information.

The third concern relates to the connection between MCMC/DP and activities in the courses. Moodle has a feature to connect competencies not only to courses but also to activities, as shown in Figure 8. Again, this might be troublesome for faculty members, as they must connect each activity to each competency one by one.
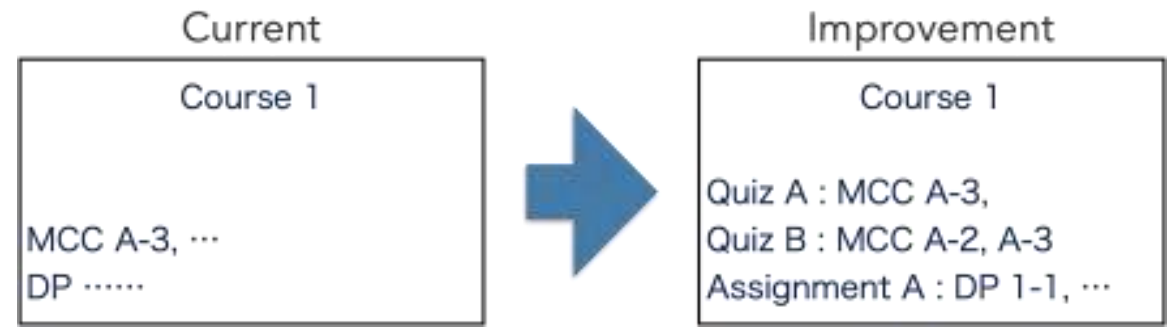

Figure 8: The future tasks to connect activities to MCC and DP

The fourth concern is regarding the input system. Because the e-syllabus has a database structure, there are some limitations to data management. For example, changing the order of classes is difficult because the data must include information such as the number of classes or the date of lectures. Moreover, the data should be exportable to make a paper-based syllabus. Although almost all data are in Moodle, paper-based syllabi are also needed in the university since some faculty members still use paper-based media. However, many teachers had started using Moodle and uploaded resumes to it because of the COVID-19 pandemic. Thus, there are some advantages of Moodle-based e-syllabi over paper-based syllabi in terms of integrating learning materials into one place.

Fifth, the DP selection interface should be addressed, as discussed previously. As noted in 5.2 , the top DP categories were selectable at the time of data collection. This should be changed to prevent selection of both top categories and subcategories.

A sixth consideration relates to the course taxonomy. In this study, classes were categorized by the syllabus domains L1 to L9. This classification is effective to analyze the macro connection between the whole JMU curriculum and MCCs/DPs, and all classes have been classified according to the syllabus categories. Detailed and micro discussions, such as investigating the connection between the Year 1 curriculum and MCCs, are needed. Yet, the current syllabus taxonomy is insufficient for such analyses. One idea for improvement is to add year information to the course ID number. Since the current id number follow the format "2019_M_L1262-2," a simple solution is placing year information after the M (e.g., "2019_M1_L1262-2"). This improvement will enable a more detailed analysis of the connection between the current curriculum and $\mathrm{MCCs} / \mathrm{DPs}$, which is essential for improving the curriculum. 


\section{Conclusion}

This study shows how to develop a Moodle-based e-syllabus for conducting curriculum mapping and competency assessments. MCC mapping revealed that some competencies were not connected to any classes. Moodle can connect e-syllabi to curriculum information and learning contents - one advantage of e-syllabi over paper-based syllabi. Although some areas in the Moodlebased e-syllabus require improvements (e.g., the user interface and connections between learning activities and competencies), the platform will be useful for IR in medical education.

\section{Acknowledgement}

This work was supported by "JSPS KAKENHI" (Grant Number JP18K02865) and "MHLW Grant” (Project Number H30-ICT-04).

\section{References}

[1]Basic Medical Education WFME Global Standards for Quality Improvement, http://wfme.org/standards/bme/ (Accessed Dec. 8, 2020)

[2] Y. Asada, "The trend of institutional research in Japanese medical education: a case study from Jichi Medical University," International Journal of Institutional Research and Management, Vol. 3, No.2, 2019, pp. 1-18.

[3] Model Core Curriculum for Medical Education in Japan, AY 2016 Revision (in English), http://www.mext.go.jp/component/a_menu/education/detail/_icsFiles/afieldfile/2018/06/ 18/1325989_30.pdf. (Accessed Dec. 8, 2020)

[4] Moodle.org, https://moodle.org/ (Accessed Dec. 8, 2020)

[5] manaba, https://manaba.jp/ (Accessed Dec. 8, 2020)

[6]Configurable Reports, https://moodle.org/plugins/block_configurable_reports (Accessed Dec. $8,2020)$

[7] Y. Asada, M. Yagi, "Moodle for Learning Analytics and Institutional Research: Exporting Data via SQLs and Plugins," International Journal of Institutional Research and Management, Vol. 4, No.2, 2020, pp. 30-43.

[8] M. Saqr, U. Fors, M. Tedre, "How learning analytics can early predict under-achieving students in a blended medical education course," Medical Teacher, Vol. 39, No. 7, 2017, pp. 757767.

[9] Y. Asada, H. Okazaki, N. Sata, H. Kawahira, S. Yamamoto, and Y. Matsuyama, "A Moodlebased e-syllabus for institutional research: curriculum Mapping and Competency Assessments," Proc. 9th IIAI International Congress on Advanced Applied Informatics (IIAI-AAI), 2020, pp. 312-315.

[10] Database activity, https://docs.moodle.org/38/en/Database_activity (Accessed Dec. 8, 2020) 
[11] SetMoodlePlainTextEditMode.user.js, https://gist.github.com/jcchurch/7e6265ce1a77ab9ab604 (Accessed Dec. 8, 2020)

[12] B. Nielsen, "Competency-based education features in Moodle," Proceedings of MoodleMoot Japan 2019 Annual Conference, pp. 6-18.

[13] I. Ko, H. Chang, "Interactive Visualization of Healthcare Data Using Tableau," Healthcare Informatics Research, Vol. 23, No. 4, 2017, pp. 349-354.

[14] Online learning, teaching and education continuity planning for schools, https:// www.ibo.org/globalassets/news-assets/coronavirus/online-learning-continuity-planningen.pdf (Accessed Dec. 8, 2020)

[15] Moodle App, https://moodle.com/app/ (Accessed Dec. 8, 2020) 